\title{
POLARITY RELATED PROBLEMS IN GROWTH OF GaN HOMOEPITAXIAL LAYERS
}

\author{
M. Leszczyński*, P. Prystawko, A. Śliwinski, T. Suski, \\ E. Litwin-Staszewska, S. Porowski
}

High Pressure Research Center, Sokołowska 29/37, 01-142 Warsaw, Poland

\section{R. Paszkiewicz, M. Teaczala}

Institute of Electronics Technology, Wrocław Technical University Janiszewskiego 11/17, 50-372 Wrocław, Poland

B. Beaumont, P. Gibart

CRHEA-CNRS, Sophia Antipolis, 06960-Valbonne, France

\author{
A. BARSKI, R. LANGER
}

Commissariat à l'Energie Atomique/Departement de Recherche Fondamentale sur la Matière Condensée, 38054 Grenoble, Cedex, France

\section{W. KNAP AND E. FRAYSSINET}

Université de Montpellier II, Groupe d'Etudes des Semiconducteurs

Place E. Bataillon, 34095 Montpellier, France

Homoepitaxial layers of GaN were grown by metalorganic chemical vapour deposition on single crystals obtained by high-pressure, high-temperature technology. For each metalorganic chemical vapour deposition run, four samples were placed, (00.1) and (00.1) faces of the Mg-doped insulating and undoped highly-conductive substrates. The layers were examined using $\mathrm{X}$-ray diffraction, photoluminescence and far-infrared reflectivity. It was found that the (00.1) easier incorporates donors resulting in higher free-electron concentrations in the layers grown on these sides of the crystals, both, undoped and $\mathrm{Mg}$-doped.

PACS numbers: $61.72 . \mathrm{Vv}, 65.70 .+\mathrm{y}$

*e-mail: mike@unipress.waw.pl 
Different incorporation of dopants onto different crystallographic faces of the $\mathrm{A}^{\mathrm{III}}-\mathrm{B}^{\mathrm{V}}$ compounds has been reported in a number of papers [1-3]. It was found that for the zinc blende structure donors are easier incorporated in the (111)B face than in the (111)A face. In our experiments we use (00.1) and (00.1) oriented hexagonal plates (wurtzite structure) of gallium nitride. Those orientations correspond to (111)A and (111)B orientations in the zinc blende structure, respectively.

For gallium nitride, the polarity-related effects have been observed in the following phenomena:

(i) heteroepitaxial layers on sapphire and homoepitaxial layers grown on the (00.1) Ga-face exhibit a much better morphology and narrower luminescence lines in comparison with the $(00.1) \mathrm{N}$-face. [4],

(ii) (00.1) faces of the undoped, highly conductive bulk crystals are chemically inert, whereas the opposite side is easily etched [5],

(iii) the morphology of as-grown single crystals is different on both sides. For the undoped, highly conductive crystals, the (00.1) faces are rough, but contain less oxygen and free electrons with respect to the flat $(00.1)$ faces [6]. For $\mathrm{Mg}$-doped insulating crystals, the rough surface is chemically active and the flat surface is inert.

The aim of the present paper is to report on the experimental results obtained for epitaxial layers grown on two opposite sides of the GaN single crystals in the same runs. We used two kinds of the substrates in order to check whether the homoepitaxial layers grown on the same kind of surface (active or inert) have the same properties.

The substrates were grown at about $15 \mathrm{kbar}$ and $1800 \mathrm{~K}$ from the nitrogen solution in gallium by a self-seeding process [7]. The active sides of the crystals were prepared by chemical-mechanical polishing, whereas the inert sides were prepared by mechanical polishing followed by the reactive ion etching which removed the layer damaged by polishing.

The layers were grown by metalorganic chemical vapour deposition (MOCVD) in the vertical reactors using TMG as a source of gallium and $\mathrm{NH}_{3}$ as a source of nitrogen. The layers were then characterized by X-ray diffraction (XRD), photoluminescence (PL), far-infrared reflectivity (FIR) and secondary ion mass spectroscopy (SIMS).

Figure 1 shows the X-ray Bragg peaks of 00.4 reflection for four samples grown in the same run, but on two opposite sides of the undoped and $\mathrm{Mg}$-doped substrates. Accordingly to the Bragg law, the angles can be inversely scaled to the lattice parameters $c$ of the layers and substrates. It can be seen that the undoped substrates are expanded due to a high free-electron concentration (about $5 \times 10^{19} \mathrm{~cm}^{-3}$ ) in comparison with the $\mathrm{Mg}$-doped insulating substrates. The layers grown on the inert sides have smaller lattice parameters with respect to the layers grown on the active sides. It should be noted that there is an additional shift of the peak for the layer grown on the inert undoped substrate. This shift originates from the tensile strain (the lattice parameters $a$ are the same for the substrate and the layer). 

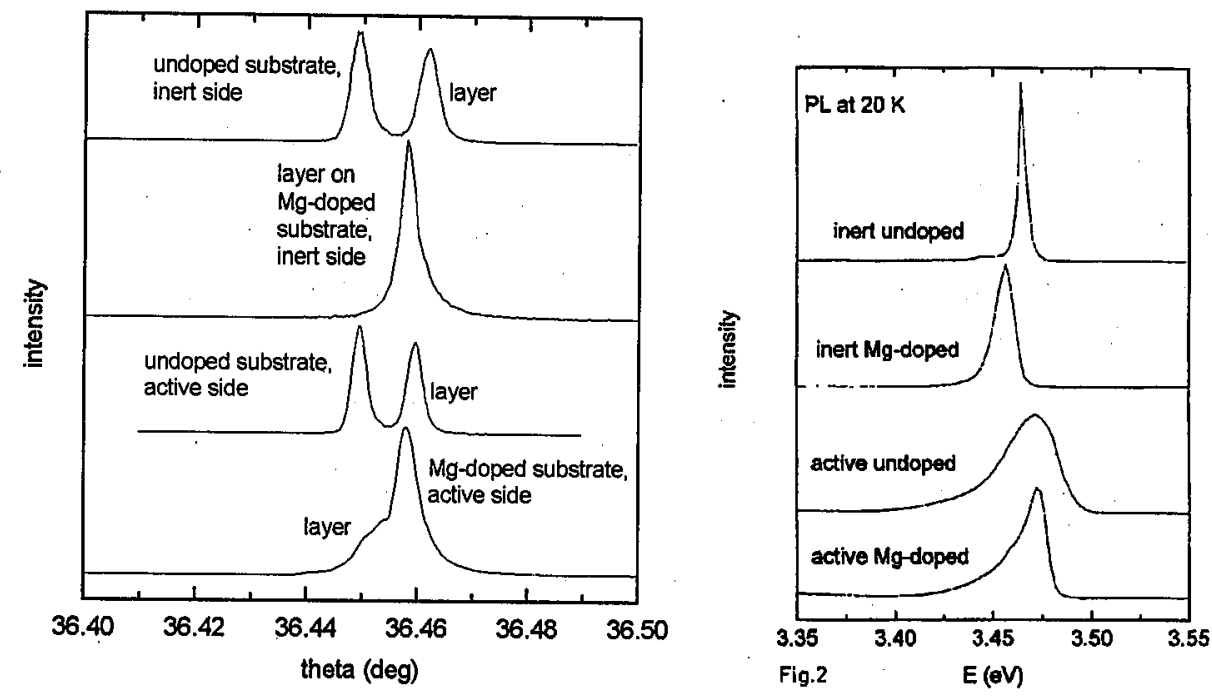

Fig. 1. Bragg peaks for the homoepitaxial layers grown on $\mathrm{GaN}$ single crystals.

Fig. 2. Photoluminescence peaks for the homoepitaxial layers grown on GaN single crystals.

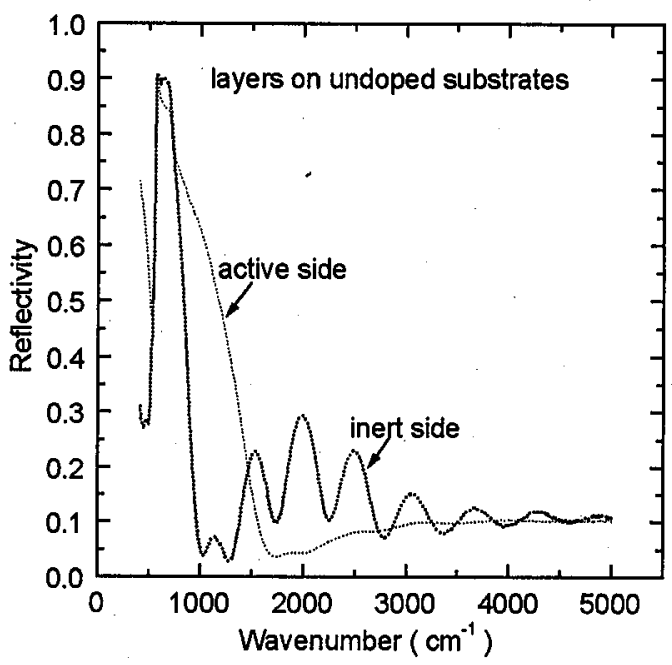

Fig. 3. Far infrared reflectivity curves for two GaN homoepitaxial layers grown on two different faces of the bulk crystal.

Figure 2 shows the PL spectra for the same samples as examined using $\mathrm{X}$-ray diffraction. The peaks for the layers grown on the inert sides are always narrower and less blue-shifted by Burnstein-Moss effect which indicates a smaller free-electron concentration with respect to the layers grown on the inert sides. The position of the peaks depends also on the strain, which should induce a red shift for the layer grown on conductive substrate. Therefore, the blue shift of the 
peak for this sample with respect to the layer grown on $\mathrm{Mg}$-doped substrate is not understood.

Finally, the higher free electron concentration for the layer grown on the inert side of the undoped crystal is confirmed by FIR (Fig. 3). It can be seen that the plasma edge for the layer on the inert side is positioned at lower energies. Additionally, the difference in the refractive index between the highly conductive substrate and this layer of much smaller free-electron concentration causes oscillations, not present for the layer on the active side.

In conclusion, there are two messages originating from the presented data:

(i) for $\mathrm{GaN}$, the incorporation of donors is easier on the (00.1) face with respect to the opposite one, which is in accordance with the data for the zinc blende (111) $\mathrm{A}^{\mathrm{III}}-\mathrm{B}^{\mathrm{V}}$ compounds,

(ii) the similar properties of the layers grown on the inert sides of the conductive and $\mathrm{Mg}$-doped crystals (the same applies for the active sides) indicate that the polarity of these two kinds of the substrates is the same with respect to their chemical properties.

The SIMS measurements performed so far have not given a univocal conclusion which donor (oxygen or nitrogen vacancy) may be responsible for the high unintentional doping of the active side.

The work was partially supported by the grant of the Committee for Scientific Research (Poland) No. PBZ 28.11/P3. M.L. is indebted to the French Ministry of Education (Centre International des Étudiants et Stagiaires) for sponsoring his research in France.

\section{References}

[1] M. Kondo, C. Anayama, N. Okada, H. Sekiguchi, K. Domen, T. Tanahashi, J. Appl. Phys. 76, 914 (1994).

[2] R. Bhat, C. Caneau, C.E. Zah, M.A. Koza, W.A. Bonner, D.W. Hwang, S.A. Schwarz, S.G. Menocal, F.G. Favire, J. Cryst. Growth 107, 772 (1992).

[3] C. Anayama, H. Sakiguchi, M. Kondo, H. Sudo, T. Fukushima, A. Furuya, T. Tanahashi, Appl. Phys. Lett. 63, 1736 (1993).

[4] K. Pakuła, A. Wysmołek, K.P. Korona, J.M. Baranowski, R. Stępniewski, I. Grzegory, M. Boćkowski, J. Jun, S. Krukowski, M. Wróblewski, S. Porowski, Solid State Commun. 97, 919 (1996).

[5] J.L. Weyher, S. Müller, I. Grzegory, S. Porowski, J. Cryst. Growth 182, 17 (1997).

[6] M. Leszczyński, G. Teisseyre, T. Suski, I. Grzegory, M. Boćkowski, J. Jun, S. Porowski, K. Pakuła, J. Baranowski, J.M. Foxon, T.S. Cheng, Appl. Phys. Lett. 69, 73 (1996).

[7] S. Porowski, I. Grzegory, J. Jun, in: High Pressure Chemical Synthesis, Eds. J. Jurczak, B. Baranowski, Elsevier, Amsterdam 1989, p. 21. 